\title{
Uso de aplicativos como tecnologia assistiva de comunicação no ambiente educacional
}

The using of apps as assistive technology in educational environment

MENDES, Edilene; Graduanda; Universidade Federal de Pernambuco

edilene.mendes.silva@gmail.com

BENEVIDES AMORIM, Fabiana; Graduanda; Universidade Federal de Pernambuco

fbamorim09@gmail.com

BATISTA, Lisandra; Graduanda; Universidade Federal de Pernambuco

lisandra.btista@gmail.com

BARBOSA, Gabryelle; Graduanda; Universidade Federal de Pernambuco

gabbebarbosa@gmail.com

NAKAYAMA, Gabriela; Doutoranda; Universidade Federal de Pernambuco

gabrielaynakayama@gmail.com

MARTINS, Laura; Doutora; Universidade Federal de Pernambuco

bmartins.laura@gmail.com

\section{Resumo}

Este artigo apresenta uma reflexão sobre o potencial do uso de aplicativos para possibilitar ou otimizar a comunicação de pessoas com deficiências ou com restrições de fala no ambiente escolar. O objetivo foi verificar a eficiência desses artefatos com o foco na habilidade comunicacional e na perspectiva do ambiente de ensino. Foram adotados os aplicativos ProDeaf e Proloquo2Go como objetos de estudo, e avaliados a partir de adaptações das ferramenta metodológica de Nakayama (2016) e dos princípios do design universal do Center for Universal Design (1997).

Palavras Chave: Design Universal; Tecnologia Assistiva; Aplicativos para comunicação.

\section{Abstract}

This article presents a reflection on the potential of using applications to enable or optimize the communication of people with disabilities or with speech restrictions in the school environment. In order to verify the efficiency of these artifacts with the focus on the communicational ability and perspective of the teaching environment. The ProDeaf and Proloquo2Go applications were adopted as study objects, and evaluated using adapted versions of the Nakayama (2016) and The Center for Universal Design (1997) analysis tools.

Keywords: Universal Design; Assistive Technology; Communication apps. 


\section{Introdução}

Os ambientes de aprendizado são fundamentados essencialmente pela comunicação, gerando compreensão mútua e efetiva entre todos os envolvidos. Imparidade nos níveis de comunicação, causados ou relacionadas à deficiências sensoriais, físicas ou cognitivas, podem representar obstáculos no processo de ensino, que é prejudicado ou se perde.

Quando um estudante apresenta um ou mais impedimentos seja na fala, audição, visão ou de mobilidade física, ações básicas do cotidiano escolar podem ser comprometidas. Fazer uma pergunta em sala de aula, ouvir a explicação do professor, tomar notas do conteúdo passado ou responder exercícios e provas escritas são só alguns exemplos de como limitações comunicacionais podem interferir no processo de aprendizagem em sala de aula. Nesses casos, se apresenta a necessidade de meios alternativos de comunicação como forma de aprendizado, tendo em vista que essas alternativas podem trabalhar como adaptações ou auxiliadores, que buscam situar de maneira equivalente a comunicabilidade para todos os envolvidos no processo. Hoje existem aplicativos para dispositivos móveis que visam estabelecer esse auxílio à comunicação desses públicos, podendo então, servir de suporte em ambientes escolares.

Tecnologias vêm sendo desenvolvidas visando conectar cada vez mais as pessoas, porém ainda não são exploradas adequadamente no ambiente educacional brasileiro. Não só por questões financeiras, pois assim como o ProDeaf há softwares gratuitos, mas também pelo sistema educacional vigente, que não estimula o uso das novas tecnologias em salas de aula. Tornando as barreiras da comunicação ainda maiores.

Os objetos de estudo são dois aplicativos para dispositivos móveis distintos, os quais apresentam propostas similares como Tecnologia Assistiva na área de comunicação interpessoal para Pessoas com Deficiência (PCD). Enquanto que aplicadas de maneiras distintas, ambas se mostram promissoras para o uso em ambientes educacionais.

Esses os dois aplicativos foram submetidos a análises para que se avaliassem suas características e possibilidades de uso dentro de princípios ergonômicos, que estudam a relação entre o usuário e os elementos do sistema, valorizando o conforto, a segurança e a eficiência da ação a ser desempenhada; e dos princípios do Design Universal, tendo em vista sua importância para garantir o uso de forma acessível e equitativa à variados usuários com capacidades diversas. Para a aplicação desses princípios às análises, foram utilizadas adaptações das ferramentas de Nakayama (2016) e do Centro de Design Universal (1997).

É esperado que seja possível entender os pontos positivos e negativos desses mecanismos como tecnologia assistiva na perspectiva de um contexto educacional.

\section{Fundamentação Teórica}

Progressivamente, aplicativos são empregados para que ampliem a eficiência do trabalho e da vida cotidiana. Eles estão presentes durante as horas de estudo e lazer; no suporte a mobilidade urbana através de apps que rastreiam o transporte público e de caronas coletivas; além do frequente uso de redes sociais e feeds de notícias. 
"Para as pessoas sem deficiência a tecnologia torna as coisas mais fáceis. Para as pessoas com deficiência, a tecnologia torna as coisas possíveis". (RADABAUGH, 1993). Devido a esse amplo leque de possibilidades e com a viabilidade de desenvolvimento de aplicativos para esses dispositivos, é necessário pensar em dispositivos como tecnologia assistiva, para promover a independência e a inclusão das pessoas com deficiência.

\subsection{Tecnologia Assistiva (TA) e Comunicação}

"Tecnologia Assistiva - TA é um termo ainda novo, utilizado para identificar todo o arsenal de recursos e serviços que contribuem para proporcionar ou ampliar habilidades funcionais de pessoas com deficiência e conseqüentemente promover vida independente e inclusão". (BERSCH \& TONOLLI, 2006).

O TA é um tema abrangente e multidisciplinar, que envolve áreas de conhecimento que compreendem estratégias, recursos, serviços e produtos. Tais recursos proporcionam e/ou ampliam habilidades funcionais para PcD, tendo como macro objetivo incentivar a independência, inclusão social e melhorar a qualidade de vida das mesmas.

A Comunicação Aumentativa e Alternativa (CAA) é destinada a atender pessoas sem fala ou escrita funcional ou em defasagem entre sua necessidade comunicativa e sua habilidade em falar e/ou escrever. São utilizados recursos como as pranchas de comunicação (Figura 1), feitas com sistemas de simbologia gráfica, letras ou palavras escritas para que o usuário da CAA possa expressar suas questões, sentimentos, entendimentos, entre outros. O suporte dessas pranchas podem ser de baixa tecnologia como pastas, álbuns, cardápio ou de alta tecnologia como softwares, sintetizadores de voz ou smartphones (Figura 2).

Figura 1 - Prancha dinâmicas de comunicação impressas

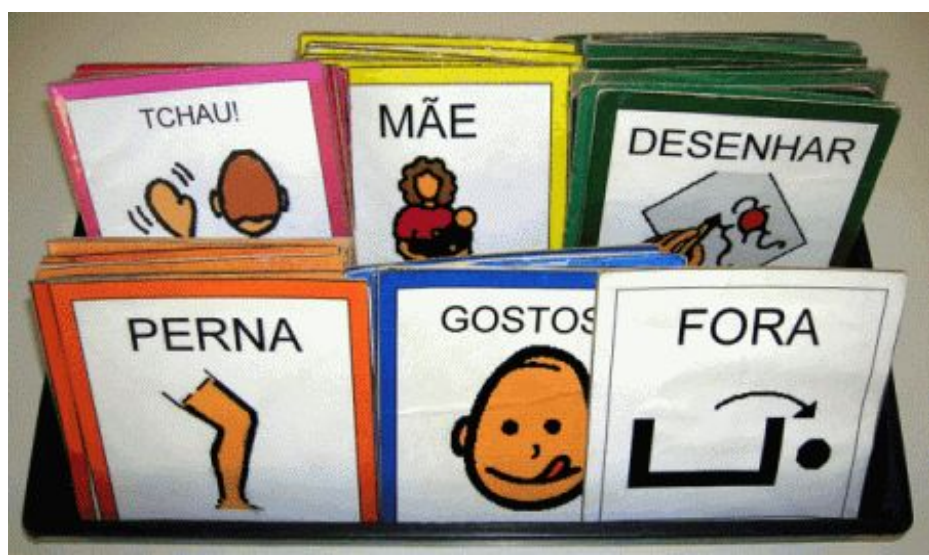

Fonte: Website do Assistiva 
Figura 2 -Prancha de comunicação digital

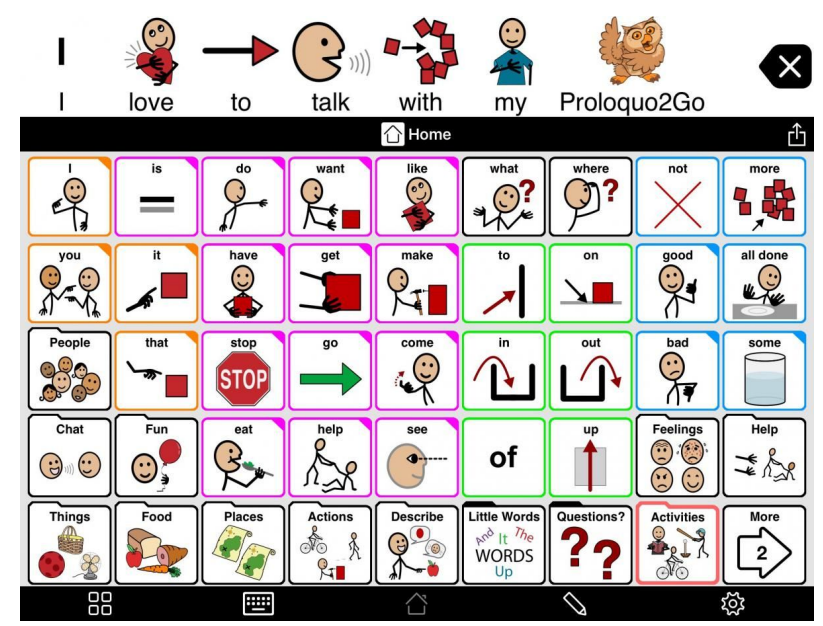

Fonte: Website do Assistive Ware

Existem Auxílios para pessoas com surdez ou com déficit auditivo que se caracterizam por incluírem mecanismos variados (infravermelho, FM). Eles podem ser aparelhos para surdez, sistemas com alertas táctil-visual, softwares que transformam em voz o texto digitado no celular e a mensagem falada em texto. Além funções como o sistema de legendas (closed-caption/subtitles), tem soluções como livros, textos e dicionários digitais em língua de sinais (Figura 3).

Figura 3: Aplicativo tradutor de LIBRAS.

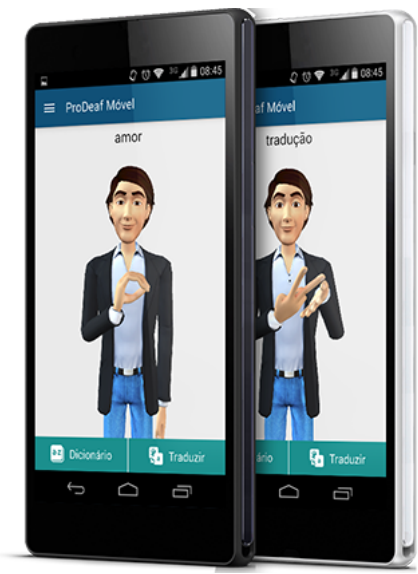

Fonte: Website do ProDeaf

\subsection{Comunicação inclusiva no ambiente escolar}

Antes de qualquer análise, é necessário refletir sobre o contexto que estamos estudando e para isso, foi realizada uma síntese acerca da história da educação. De acordo com Coutinho (2005), a história da educação começa desde a origem do próprio homem. Na era primitiva, 
baseava-se somente à prática, e o conhecimento era passado para a sobrevivência do homem, através da oralidade e exemplos práticos.

O modelo educacional desde então era hierarquizado e autoritário, apenas em meados do século XIX, ele passa a ser questionado pelos educadores que reivindicam a participação ativa dos alunos no processo de aprendizagem. E assim, resgataram princípios da educação onde também se valorizam as experiências vivenciadas pelo aluno.

A educação nem sempre atendeu à todos, pois a exclusão esteve sempre presente em sua trajetória, seja ela social, racial, de gênero, ou a presença de alguma deficiência. Essas diferenças eram usados como parâmetros para definir a quantidade e a qualidade do processo didático individual. O curso da exclusão educacional pode ter sido amenizado ao longo dos anos, mas ainda é bastante presente, principalmente em países subdesenvolvidos em que não existe investimento significativo na educação.

Entre os excluídos estão as pessoas com deficiência, pois, a partir do momento que estabelecemos um padrão de normalidade, estamos automaticamente negando as diferenças, e rotulando pessoas. Através dos anos, educadores e movimentos que lutam pela inclusão das pessoas com deficiência, têm transformado o modo de pensar educação.

Há vários aspectos e condições que devem ser melhorados e implementados para que de fato haja a inclusão escolar, e não apenas a integração desses alunos às práticas regulares de ensino. Fatores como infraestrutura adaptada, qualificação de professores especializados, material didático adequado e, um dos fatores mais importantes para um ambiente de ensino inclusivo, o qual é investigado neste artigo: a comunicação. Fator essencial no desenvolvimento do aluno, tanto pela contínua comunicação com os outros alunos, os professores e vice-versa, bem como para promover sua autonomia e independência.

Um exemplo de investimento na solução do problema de inclusão comunicativa foi a escola Milford, no estado de Connecticut, EUA, que de acordo com uma matéria feita pela $A B C$ News, em 2011, comprou 3 ipads para os 3 estudantes autistas. Eles argumentam que é uma solução mais barata, e que tem ajudado crianças com dificuldades de comunicação, pois oferece uma experiência mais interativa. Aos poucos foram percebendo o quão útil os aplicativos eram para o aprendizado dessas crianças. Então, foi visto que existe uma possibilidade prática de inclusão por meio dessas tecnologias e isso representa um potencial para a pessoa com deficiência.

\subsection{Design Universal}

Segundo The Center for Universal Design (1997) o design universal consiste no planejamento de produtos que visa atender a maior gama de usuários possível, segundo suas características antropométricas, biomecânicas e sensoriais, independente do público-alvo ao qual o produto se destina. O produto, desta forma, deve ser acessível para toda uma gama de capacidades ou habilidades sem deixar de lado as diferenças culturais, sociais e econômicas, além de ser projetado evitando a existência exclusiva de produtos especiais para pessoas com deficiências e restrições.

No design, seu objetivo é tornar a interação com produtos, sistemas ou ambientes o mais 
eficiente possível, realizando seus estudos e projetos por meio da promoção e análise dos seguintes princípios do Center for Universal Design (1997) (tradução das autoras):

- Uso equitativo - útil e que atenda a um público com habilidades diversas.

- Uso flexível - que permita que sejam aplicadas preferências e habilidades individuais dos usuários.

- Uso simples e intuitivo - que seja de fácil compreensão, independente de conhecimentos e experiências anteriores, habilidade específica ou nível de concentração do usuário.

- Informação perceptível - que seja suficientemente informativo, independente de condições do ambiente de uso ou de habilidades sensoriais do usuário.

- Tolerância a erros - que minimize e previna riscos e consequências adversas de uso, sejam as mesmas acidentais ou não intencionais.

- Mínimo esforço físico - que seja efetivo e confortável, gerando o mínimo de esforço em seu uso.

- Tamanho e espaço para aproximação e uso - que tenha tamanho e espaço apropriados para aproximação, uso e alcance, independente da postura, estatura e mobilidade do usuário.

\section{Metodologia}

Para o presente trabalho foi desenvolvido um levantamento bibliográfico da literatura relevante aos temas de inclusão, educação, tecnologia assistiva e deficiências, a fim de se estabelecer os conceitos e teorias fundamentais para o desenvolvimento das análises propostas. Os objetos de estudo foram os aplicativos ProDeaf e Proloquo2Go, sendo eles avaliados a partir de adaptações das ferramentas metodológicas de Nakayama (2016) e dos princípios do design universal do Center for Universal Design (1997).

Durante o grupo de estudos "Design universal, Acessibilidade e Inclusão" ministrado pela Profa. Drä. Laura Martins e a Profá. MSc. Gabriela Nakayama no Bacharelado em design da Universidade Federal de Pernambuco, foram realizadas as análises de produtos desenvolvidos para auxiliar o dia a dia de usuários com dificuldades e/ou deficiências, sendo alguns disponíveis no mercado.

Dentre os produtos, foi identificado nos aplicativos ProDeaf e no Proloquo2Go um ponto em comum, ambos estão presentes como facilitadores na comunicação de pessoas com ou sem deficiência ( $P C D)$ entre si. Apesar de suas diferenças, foi considerado que eles têm um grande potencial como tecnologia assistiva a serem usadas dentro das salas de aula.

A presença dos dois aplicativos estudados denota o estudo de caso comparativo, onde cada análise é feita sob os mesmo parâmetros para cada objeto de estudo. Os resultados das partes então são contrapostos e as conclusões das análises são, então, construídas.

\subsection{Ferramentas de análise}


As tabelas disponibilizadas aos alunos visavam analisar o artefato e seu uso, tendo sido a primeira desenvolvida por Nakayama (2016) e a segunda desenvolvida por The Center for Universal Design (1997), ambas adaptadas para uso e adequação em sala de aula.

A primeira tabela (figuras 4 e 5) de Nakayama (2016) consiste em seis requisitos, sendo cada um deles detalhado em alguns pontos. São eles os requisitos norteadores, gerais, dos materiais, de uso, avaliativos e finais do produto. Para cada ponto analisado é necessário classificar o artefato em uma escala de avaliação, colocando "atende", "não atende" ou ainda "não se aplica". É possível também justificar a avaliação dada.

A segunda tabela é de análise de uso do artefato do The Center for Universal Design (1997). Ela é baseada nos 7 (sete) princípios do design universal, tendo assim 7 tópicos de "princípios e regras" para avaliar detalhadamente produtos através de pontos específicos. A aplicabilidade dos princípios e regras no artefato em questão é avaliada em uma escala de 0 a 5 , onde 0 é "não se aplica", 3 "aplicável" e 5 "muito aplicável", e também através de justificativas quanto a classificação.

Por se tratar de análises feitas em equipe, para ambas tabelas foram utilizados os mesmo métodos de preenchimento. Cada pessoa analisou o artefato individualmente e marcou nas tabelas a letra inicial de seu nome, a equipe então se reunia e debatia os resultados. Quando haviam pontos discordantes, era aderido a melhor justificativa para então gerar uma tabela final.

Figura 4 - Tabela de análise do artefato do Proloquo2go

PROLOQUO2GO

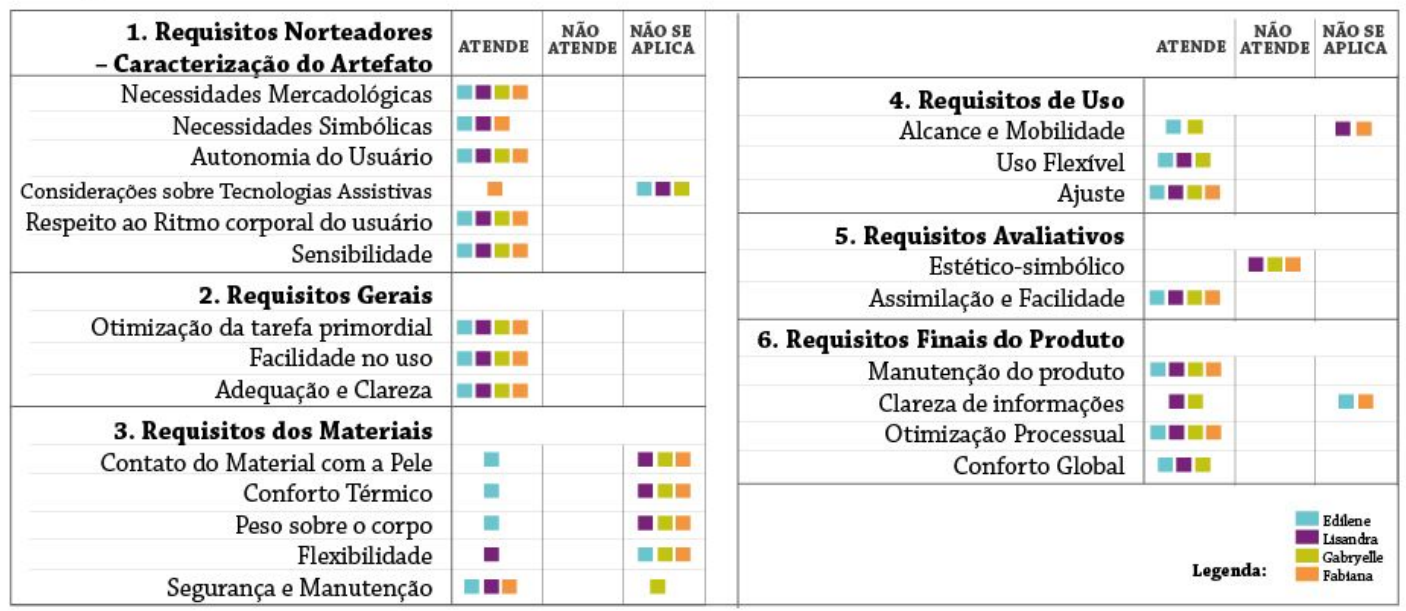

Fonte : Adaptado de “Nakayama” (2016)

Figura 5 - Tabela de análise do artefato do ProDeaf 


\begin{tabular}{|c|c|c|c|c|c|c|c|}
\hline $\begin{array}{l}\text { 1. Requisitos Norteadores } \\
\text { - Caracterização do Artefato }\end{array}$ & ATENDE & $\begin{array}{l}\text { NÃO } \\
\text { ATENDE }\end{array}$ & $\begin{array}{l}\text { NÃO SE } \\
\text { APLICA }\end{array}$ & & ATENDE & 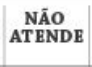 & $\begin{array}{l}\text { NÃOO SE } \\
\text { APLICA }\end{array}$ \\
\hline Necessidades Mercadológicas & En= & & & 4. Requisitos de Uso & & & \\
\hline Necessidades Simbólicas & an & & & Alcance e Mobilidade & ana & & \\
\hline Autonomia do Usuário & 물 & & & Uso Flexível & 믈 & & = \\
\hline Consideraçōes sobre Tecnologias Assistivas & 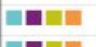 & & & Ajuste & 田 & & 물 \\
\hline Respeito ao Ritmo corporal do usuário & (a) & & & 5. Requisitos Avaliativos & & & \\
\hline Sensibilidade & (1) & & & Estético-simbólico & En & & \\
\hline 2. Requisitos Gerais & & & & Assimilação e Facilidade & 불 & & \\
\hline Otimização da tarefa primordial & Ema & & & 6. Requisitos Finais do Produto & & & \\
\hline Facilidade no uso & man & & & Manutenção do produto & a & & 물 \\
\hline Adequação e Clareza & man & & & Clareza de informaçð̃es & $\mathbf{m}=$ & & \\
\hline 3. Requisitos dos Materiais & & & & Otimizaçăo Processual & Ene & & \\
\hline Contato do Material com a Pele & & & 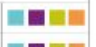 & Conforto Global & an & & \\
\hline Conforto Térmico & & & = & \multirow{4}{*}{\multicolumn{4}{|c|}{ Legenda: }} \\
\hline Peso sobre o corpo & & & 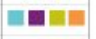 & & & & \\
\hline Flexibilidade & & & 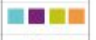 & & & & \\
\hline Segurança e Manutenção & & & $\mathbf{E}=$ & & & & \\
\hline
\end{tabular}

Fonte : Adaptado de “Nakayama” (2016)

\section{Análises}

\subsection{ProDeaf}

O ProDeaf é um conjunto de softwares capaz de traduzir o texto e voz em Português para LIBRAS (Língua Brasileira de Sinais), com o intuito de permitir a comunicação entre surdos e ouvintes. O ProDeaf possui três formas de uso que foram desenvolvidos para ampliar o acesso dos seus usuários. A primeira forma é o ProDeaf WebLibras, onde é possível que sites comprem o código e o aplique em seu domínio, assim, qualquer usuário que acesse o site pode utilizar a aplicação para traduzir o conteúdo para LIBRAS. Na segunda, o usuário instala o software que traduz os conteúdos dos sites que ele acessa em seu próprio computador. E a terceira forma é mobile( Figura 3), um aplicativo que traduz texto ou áudio fornecido pelo usuário. As versões web são gratuitas em uma quantidade limitada de traduções enquanto a versão mobile é totalmente gratuita, mas precisa de conexão com a internet.

\subsection{Proloquo2Go}

"Uma voz para aqueles que não podem falar", é como se intitula o Proloquo2Go. Um aplicativo de comunicação (Figura 6) através de símbolos que ajudam a promover o desenvolvimento da linguagem para aqueles que têm alguma dificuldade de fala. $O$ programa se enquadra como CAA (Comunicação Aumentativa e Alternativa), vocalizando a fala a partir de imagens ou palavras selecionadas pelo usuário. Segundo o desenvolvedor é indicado para crianças e adultos com paralisia cerebral, autismo, síndrome de down, AVC, entre outras causas de deficiência na fala. 
O programa funciona disponibilizando palavras separadas por categorias, cada qual com uma imagem correspondente (Figura 2). Ao tocar na palavra ou imagem, o usuário pode formar frases combinando-as, que então, são vocalizadas pelo app. Essa voz sintética pode ser escolhida pelo usuário: masculina ou feminina, adultos ou infantis também. Esse aplicativo está disponível em 3 idiomas, Inglês, Francês e Espanhol. Com um vocabulário com mais de 7000 itens, também podendo adicionar novos. Ele funciona somente na plataforma IOS, custa \$189 dólares, cerca de \$345 reais e não é necessário estar conectado à internet.

Figura 6 - Demonstração de uso do Proloquo2Go.

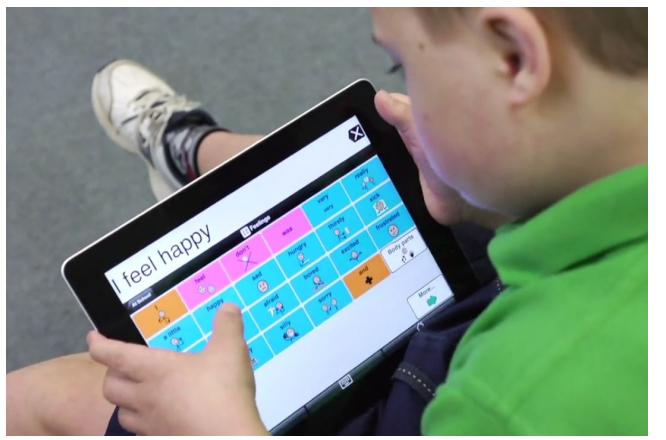

Fonte: Screenshot do vídeo do website Assistive Ware

\subsection{Análise do Artefato - Nakayama (2016)}

\subsubsection{Proloquo2Go:}

O software procura atender a necessidade de comunicação do usuário, que é essencial no local de aprendizado através do suporte e estímulo à autonomia. Essa autonomia é adquirida após um período de adaptação ao uso no qual o aluno recebe suporte para aprender a lidar com o aplicativo, seja de um professor ou de um responsável. $\mathrm{O}$ app mostra-se flexível a diferentes níveis de vocabulário (estão disponíveis para uso três níveis, do mais básico ao mais avançado). Incluindo a capacidade do app de 'aprender' e assim, se adaptar e desenvolver à medida que o usuário faz uso da ferramenta, otimizando assim a experiência. É ainda fornecido pelos desenvolvedores, suporte técnico, periódicas atualizações e a disponibilidade de diversos vídeos e materiais de instrução que visam orientar e facilitar o uso.

No entanto, tem-se em debate a satisfação da experiência estética, pois, apesar da impossibilidade de coleta de respostas diretas dos usuários relativas a agradabilidade do uso, os elementos gráficos em geral cumprem sua função de serem adequadamente identificados, de acordo com o contexto. $O$ aplicativo também deixa a desejar no sentido da organização estrutural dos elementos visuais, pois apresenta muitas informações sem devida hierarquização, podendo causar um desconforto visual indesejável para o público.

\subsubsection{ProDeaf:}

Esse artefato procura atender usuários que precisam ter acesso a conteúdos online, que a princípio se encontram apenas escritos em português, e os traduz para LIBRAS. Essa tradução se 
faz necessária para pessoas surdas que podem não ser bilíngues. $O$ uso do aplicativo atende os princípios da acessibilidade, por ter um aplicativo e um site como tradutores gratuitos. Possibilita até mil traduções gratuitas em qualquer website, se precisar mais que isso, é possível a compra de pacotes. Porém, de acordo com essa análise, é observada a limitação de traduções disponíveis e a necessidade de estar conectado à internet para usar o aplicativo, fatores que pesam pois limitam o uso.

O ProDeaf promove a autonomia do usuário, apresenta uma interface "clean" e facilmente manipulável. O app apresenta os recursos necessários para realização da atividade principal e, apenas quando necessário, os recursos adicionais .

\subsection{Análise do Uso - Center for Universal Design (1997)}

\subsubsection{ProloQuo2Go:}

Figura 7 - Tabela de análise do uso do artefato do ProDeaf

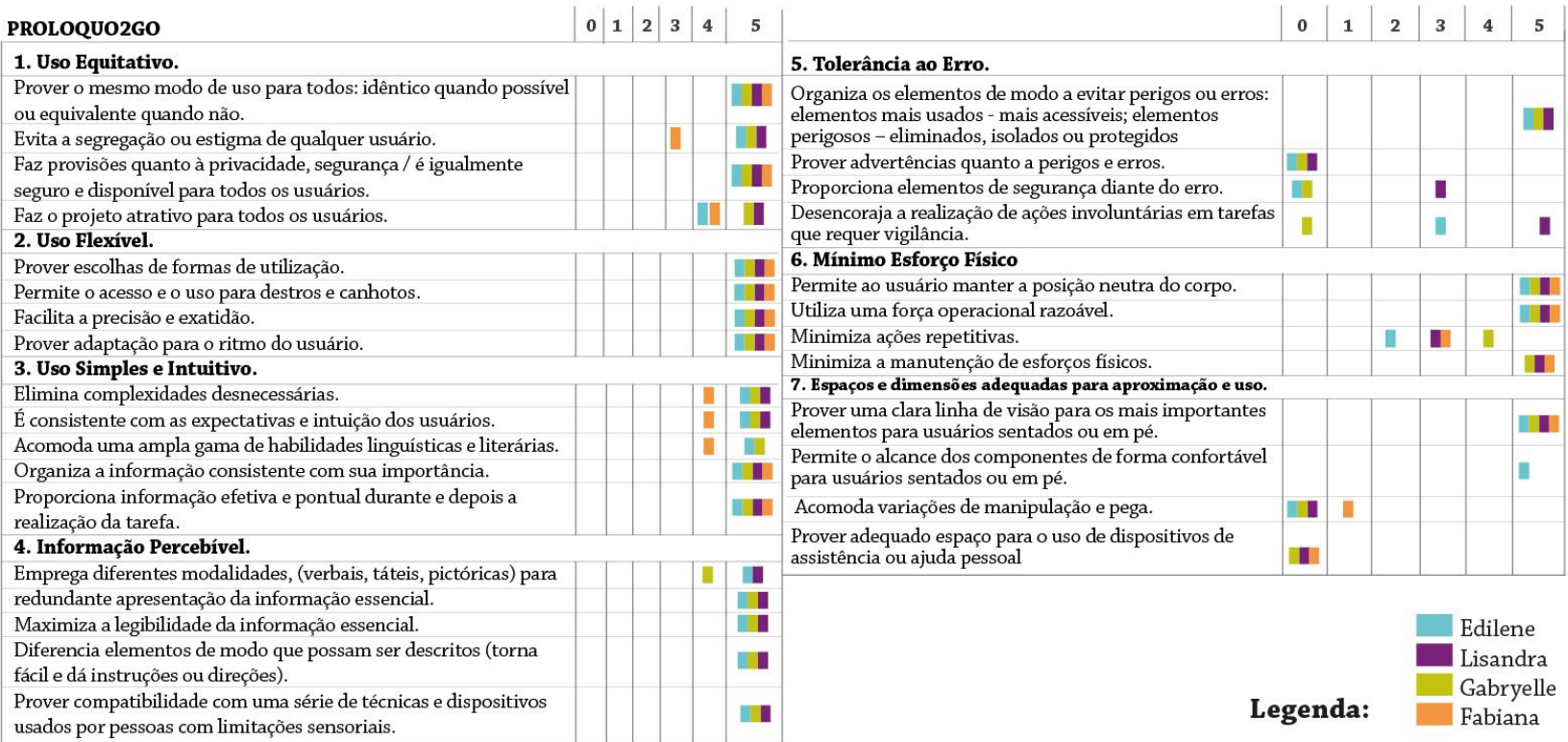

Fonte: Adaptado de "The Center for Universal Design” (1997)

O aplicativo promove o uso equitativo para qualquer usuário a partir do uso da cognição, visão e manejo fino, considerando que é necessário um domínio mínimo dessas funções, através do desempenho autônomo de capacidades como de análise, interpretação, inibição de estímulos irrelevantes para determinada tarefa, percepção visual e espacial, coordenação olho-mão e o manejo das pontas dos dedos com precisão e relativa rapidez. Dessa forma, mesmo que com diferentes níveis de vocabulário os usuários conseguem, através do aplicativo, estabelecer a comunicação com o próximo.

O ProloQuo2Go permite o uso flexível via as opções de uso trilíngue do aplicativo, dentro dos quais ainda estão disponíveis dialetos e diferentes vozes sintéticas que variam em gênero, 
idade e entonação, permitindo que o usuário opte aquele que mais se adequa às suas preferências.

Os elementos gráficos são de grande importância para auxiliar o usuário durante o uso, logo assume-se que o acervo pictórico cumpre bem sua função de representar ideias, ações e palavras.

No que tange a interface do aplicativo, palavras que são frequentemente usadas, são priorizadas aparecendo mais ao centro da tela em posição de maior destaque e de maneira acessível. $O$ aplicativo faz uso de diferentes possibilidades de representações da linguagem, entre as modalidades pictórica, verbal/numérica e esquemática. Dessa forma, englobando e tornando as informações redundantes, de maneira que auxilia o usuário, através da repetição e reforço da informação.

As interfaces do aplicativo centralizam as informações de maior relevância, e o próprio sistema trabalha de forma que exista predição das palavras seguintes durante a formação das frases à medida que se usa o aplicativo. $O$ usuário faz pouco esforço físico extra para operar o aplicativo, de maneira que mesmo as frequentes ações repetitivas não o sobrecarregam.

Contudo, o produto é vendido apenas para dispositivos IOS e o preço de compra não é acessível para todos, logo parte de seu público pode ser desestimulado e até impossibilitado de adquirir o aplicativo. Existem ainda as barreiras da língua, pois o usuário deve se enquadrar como falante da língua inglesa, francesa ou espanhola para ser capaz de usar o aplicativo.

\subsubsection{ProDeaf:}

Figura 8 - Tabela de análise do uso do artefato do Proloquo2go

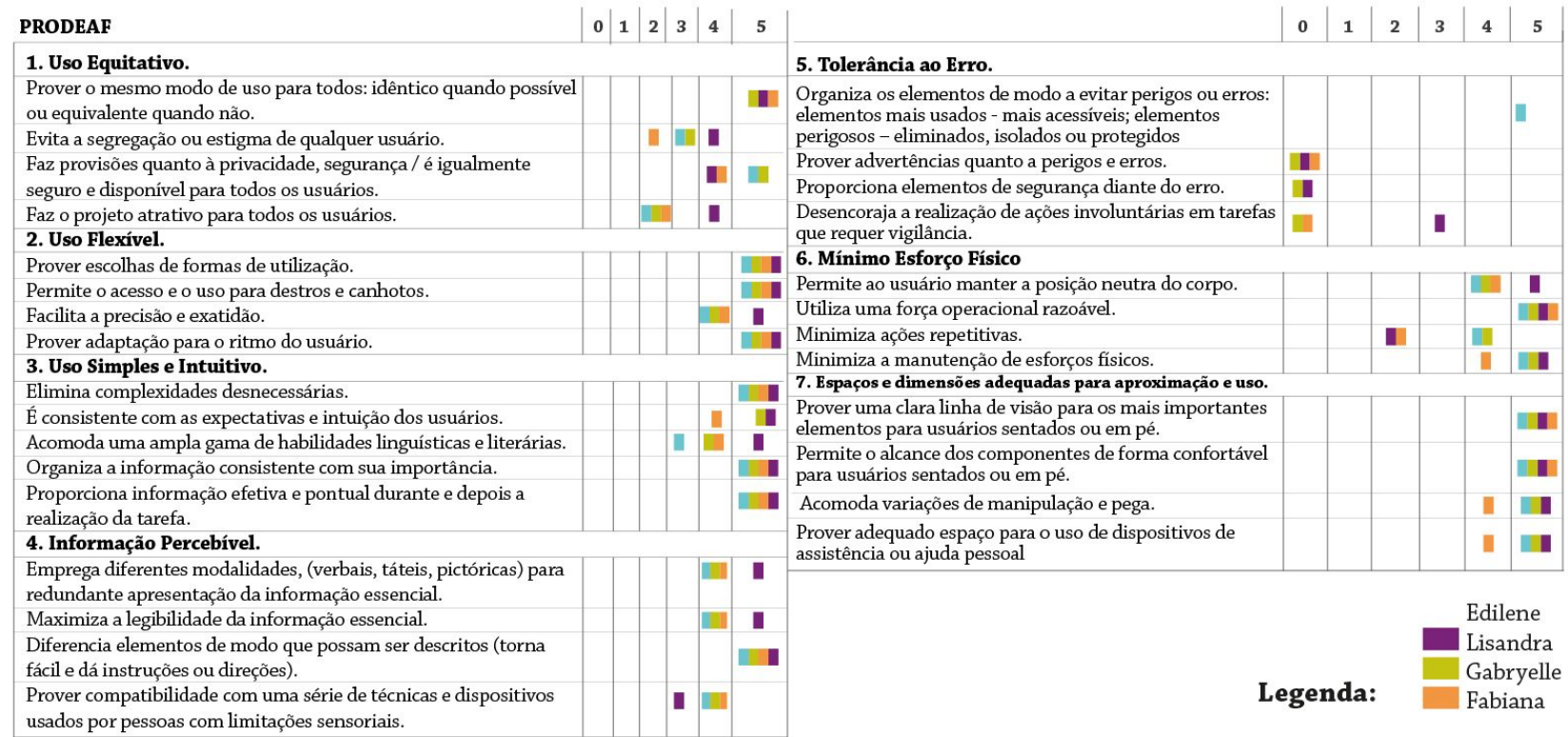

Fonte: Adaptado de "The Center for Universal Design" (1997) 
De acordo com a análise o aplicativo promove o uso equitativo para os públicos que precisam de suporte para a comunicação, sem estigmatizar, segregar e de maneira segura. O uso se mostra flexível quando se dispõe como suporte para a comunicação tanto para PcD num ambiente escolar, como para estudantes que estão aprendendo LIBRAS por exemplo, com ou sem alguma deficiência.

O uso é simplificado e a interface prioriza as informações mais relevantes. Através do emprego de informações nos formatos verbal e pictórica o aplicativo maximiza a legibilidade e é compátivel com diferentes limitações sensoriais. Os esforços físicos são mínimos, adequados ao uso de aparelhos como smartphones e tablets, de forma que é possível usar o produto em posição neutra do corpo.

No entanto, aplicativo não garante a tolerância a erros por parte do usuário, estando sujeito a má identificação e interpretação de palavras e termos. Também não está claramente disponibilizada a opção de corrigir eventuais erros cometidos durante o uso.

\subsection{Comparação}

Embora tenham o mesmo objetivo de promover a comunicação, a forma como cada aplicativo o faz é de forma diferenciada. O ProDeaf pode ser usado tanto pelo surdo, quanto pelo ouvinte. Porém, na maioria dos casos, há somente uma via de comunicação através do app, que é a do ouvinte para o surdo. Onde o ouvinte escreve ou grava sua mensagem, e o app traduz em LIBRAS, mas o contrário não acontece pois não há um leitor das formas gestuais feitas pelo surdo. Assim, o surdo só pode comunicar através de texto. E no Proloquo2Go, a comunicação é de via dupla, pois o aplicativo funciona como auxílio na comunicação da pessoa com deficiência de fala. Enquanto no ProDeaf, os usuários tanto podem ser ouvintes como surdos, no Proloquo2Go apenas pessoas com dificuldades de fala utilizam. Foi observado, por fim, que os usuários nas duas soluções são fundamentalmente diferentes em suas habilidades mas ao mesmo tempo, habitam nos momentos de ensino, o mesmo ambiente didático.

No ProDeaf podemos perceber a simplicidade das funções existentes em todas suas aplicações. Ele somente utiliza textos e gravações, e animações previamente programadas. Quanto ao Proloquo2Go, é um pouco mais complexo, pois ele faz uma combinação de palavras chaves e formam uma frase transmitidos por uma voz. Além disso, há outros recursos, como criar novas palavras e adicionar símbolos.

Devido a essa diferença de complexidade, a promoção da autonomia dos usuários ao utilizar cada aplicativo é diferente. No Proloquo2Go a curva de aprendizado é determinada pelo tempo de adaptação ao aplicativo onde o usuário aprende e se habitua ao uso. Essa habituação leva aproximadamente 6 meses. Depois disso, ele consegue utilizar sozinho, só precisando de um mediador para interferir na de seu vocabulário ocasionalmente. Já no ProDeaf, o usuário consegue utilizar todas as funções do aplicativo sem auxílio de um mediador ou manual, a partir de uma quantidade de tempo usual para conhecer o aplicativo.

\section{Considerações Finais}

Como designers, devemos estar sempre atentos ao desenvolvimento destes artefatos. E, tendo como base estudos como o aqui apresentado, buscar a criação de artefatos inclusivos e de 
usabilidade o mais eficiente possível, participando da produção, analisando e experimentando novos projetos para alcançar o melhor resultado para o uso de todo e qualquer indivíduo. É também no ambiente educacional, no entanto acadêmico, que devem surgir as primeiras discussões sobre a importância da atuação do designer nessa área, fomentando as pesquisas do design inclusivo e do design como agente otimizador de ferramentas assistivas.

Assim como vimos através da história da educação, o processo de inclusão continua se modelando, buscando as melhores formas de promover a comunicação, sendo ela a principal ferramenta do ser humano para conviver em sociedade. Devido a sua importância no meio escolar, onde são construídas as bases de formação do indivíduo, é necessário encontrar um sistema de comunicação para todos, igual quando possível e equivalente quando não.

Apesar de não terem sido conduzidos testes com usuários, foi possível enxergar no ProDeaf e no ProloQuo2Go a iniciativa para romper essas barreiras de comunicação. É preciso incentivar o uso de aplicativos para promover a interação social e educacional desses estudantes. Assim, concluímos que é possível conectar pessoas com diferentes necessidades, o que pode ocorrer de diversas maneiras, sendo a comunicação provavelmente a mais eficaz. De acordo com esse estudo, é evidente ainda a importância de serem realizadas avaliações em produtos e projetos existentes no mercado, pois, com isso é possível proporcionar maior embasamento teórico analítico para designers em seus processos projetuais. Esse reconhecimento analítico de produtos já existentes permite que o designer possa projetar mais consciente e em maior concordância com o contexto atual.

\section{Referências}

Apesar De Avanços, Surdos Ainda Enfrentam Barreiras De Acessibilidade. Disponível em: <http://www.brasil.gov.br/cidadania-e-justica/2016/09/apesar-de-avancos-surdos-ainda-enfrenta m-barreiras-de-acessibilidade> Acesso em 05 de dezembro de 2017.

A Voice For Those Who Cannot Speak: ProloQuo2Go. Disponível em: <http://www.assistiveware.com/product/proloquo2go> Acesso em 04 de dezembro de 2017.

BERSCH, R. Introdução À Tecnologia Assistiva, 2013. Disponível em: <http://www.assistiva.com.br/Introducao Tecnologia Assistiva.pdf $>$ Acesso em 04 de dezembro de 2017.

BERSCH, R.; TONOLLI, J. C. Introdução ao Conceito de Tecnologia Assistiva e Modelos de Abordagem da Deficiência, 2006. Disponível em: <http://www.bengalalegal.com/tecnologiaassistiva > Acesso em 04 de dezembro de 2017.

CARVALHO, R. E. Educação Inclusiva com os Pingos nos Is. 2. ed. Porto Alegre: Mediação, 2005. Disponível em: <https://jefersongonzalez.files.wordpress.com/2013/09/1_carvalho.pdf> Acesso em 04 de dezembro de 2017. 
História Da Educação. Disponível em:

<https://www.infoescola.com/pedagogia/historia-da-educacao/> Acesso em 04 de dezembro de 2017.

IIDA, I. Ergonomia Do Manejo: Proposição De Uma Metodologia Para Análise Do Manejo Em Ferramentas Manuais. Disponível em:

<http://bibliotecadigital.fgv.br/ojs/index.php/abpa/article/viewFile/17150/15939> Acesso em 05 de dezembro de 2017.

Marin Co. To Issue Ipads For Its Special Ed Students. Disponível em:

<http://abc7news.com/archive/8024279/> Acesso em 05 de dezembro de 2017.

O Que É Autismo Ou Transtorno Do Espectro Autista?. Disponível em:

<http://autismo.institutopensi.org.br/informe-se/sobre-o-autismo/o-que-e-autismo/> Acesso em 05 de dezembro de 2017.

ProDeaf. Disponível em: <http://prodeaf.net/> Acesso em 04 de dezembro de 2017.

RADABAUGH, M. P. NIDRR's Long Range Plan - Technology for Access and Function Research Section Two: NIDDR. Research Agenda Chapter 5: TECHNOLOGY FOR ACCESS AND FUNCTION. Disponível em <http://www.ncddr.org/rpp/techaf/Irp ov.html> Acesso em 04 de dezembro de 2017.

Síndrome De Down: O Que É. Disponível em:

<http://www.movimentodown.org.br/sindrome-de-down/o-que-e/> Acesso em 05 de dezembro de 2017.

The Center for Universal Design. The Principles of Universal Design. Versão 2.0, North Carolina University State, 1997. Disponível em:

<http://www.ncsu.edu/ncsu/design/cud/pubs_p/docs/poster.pdf>. Acesso em 05 de dezembro de 2017. 